\title{
BINOCULAR EYE MOVEMENTS DURING ACCOMMODATIVE VERGENCE
}

\author{
Robert V. Kenyon, Kenneth J. Ciuffreda and Lawrence Stark \\ School of Optometry, University of California. Berkeley, CA 94720. U.S.A.
}

(Received 24 March 1977; in revised form 4 August 1977)

\begin{abstract}
Binocular eye position was monitored by the photoelectric technique during accommodative vergence. Contrary to previous reports indicating that accommodative vergence was a uniocular phenomenon, without exception, binocular accommodative vergence movements were recorded. The total vergence amplitude in the viewing eye was reduced, on the average. by approximately $88^{\circ}$; with respect to the vergence movement measured in the covered eye. Some saccadic eye movements that occurred during vergence movements were likewise reduced in amplitude in the viewing eye by up to $20 \%$. Smooth eye movements were utilized to counteract the vergence movement in the viewing eye. This smooth movement alone, or in conjunction with a late saccade. returned the eye to the target and helped to maintain the retinal image of the target coincident with the foveal center for the duration of the accommodative vergence movement. Thus. there appears to be a fixation-holding mechanism which produced a general attenuation of both vergence and some saccadic movements in the viewing eye. Although this control strategy produced violations of Hering's law with respect to the magnitude of the movements in the eyes but not with respect to the direction of the movement. it was implemented in the interest of retaining the target within the sensitive foveal region.
\end{abstract}

\section{INTRODUCTION}

A synkinetic relationship between the human accommodative system and the vergence eye movement system was reported by Johannes Müller (1826). He observed that if one eye were covered, and the viewing eye changed fixation from a far to a near target along its line of sight, the covered eye rotated inward.

Objective measures of the dynamical characteristics of accommodative vergence have recently been made in monkey and man. In many of these studies. the position of the viewing eye was not monitored (Troelstra et al., 1964; Robinson, 1966; Brodkey and Stark, 1967: Yamamoto, 1968. 1970; Keller. 1973; Krishnan, Phillips and Stark, 1973). Keller and Robinson (1972) monitored the viewing eye in a monkey highly trained in an accommodative vergence task and did not observe movement in the eye. Pickwell (1972), using photographic and direct visual observation methods, did not observe movement in the viewing eye during accommodative vergence. The remainder of the contemporary studies on accommodative vergence recorded the position of both eyes. Allen (1949) dynamically photographed both eyes during accommodative vergence, but his discussion was restricted to the technical aspects of the instrumentation. Alpern and Ellen (1956) utilized the electro-oculographic method to record eye movements and reproduced the Müller experiment. They concluded, "One important finding of these measurements is that, for our subject at least, only the occluded eye moves," and furthermore maintained that the movement in the viewing eye was totally damped as a result of the predominance of the fixation system. Hermann and Samson (1967) used the infra-red reffection technique to determine objective clinical accommodative vergence to accommodation ratios (AC/A ratios) in patients. They stated, the position of the fixated eye is quite steady dur- ing these movements except for infrequent blinks." Thus, the conclusion of these modem workers was that the viewing eye remained stationary, and only the occluded eye moved during accommodative vergence-an apparent gross violation of Hering's law of equal innervation to corresponding muscles of the eye.

Using precision infrared photoelectric eye movement recordings, we show disjunctive movement of the viewing eye in the direction predicted by Hering's law, but markedly attenuated with respect to the magnitudes of the movements in the covered eye. Moreover, some saccadic movements that occurred during the vergence movement were likewise reduced in the viewing eye. Thus, our findings reopen important questions concerning accommodative vergence. Hering's law, and higher level control of eye movement.

\section{METHODS}

\section{Targets and target alignment}

In the design of an experiment to stimulate only the accommodation system and to observe the resultant movement of each eye through the accommodative vergence synkinetic relationship, it is imperative that all targets in visual space be precisely aligned along the line of sight of the viewing eye. Exact target alignment precludes the occurrence of eye movements unrelated to stimulation of the accommodation system.

Three targets were mounted on an 'optical bench at distances of 100,50 and $25 \mathrm{~cm}$ from the estimated center of rotation of the subject's viewing eye (Fig. 1). These target distances corresponded to accommodative stimuli of 1.0 , 2.0 and $4.0 \mathrm{D}$, respectively. The middle and near targets consisted of small lucite plates with fine crosses etched upon the front surfaces which subtended visual angles of 1.5 and $3.0^{\circ}$, respectively: the etched lines forming the crosses subtended visual angles of 2 and $4 \mathrm{~min}$ arc for the 


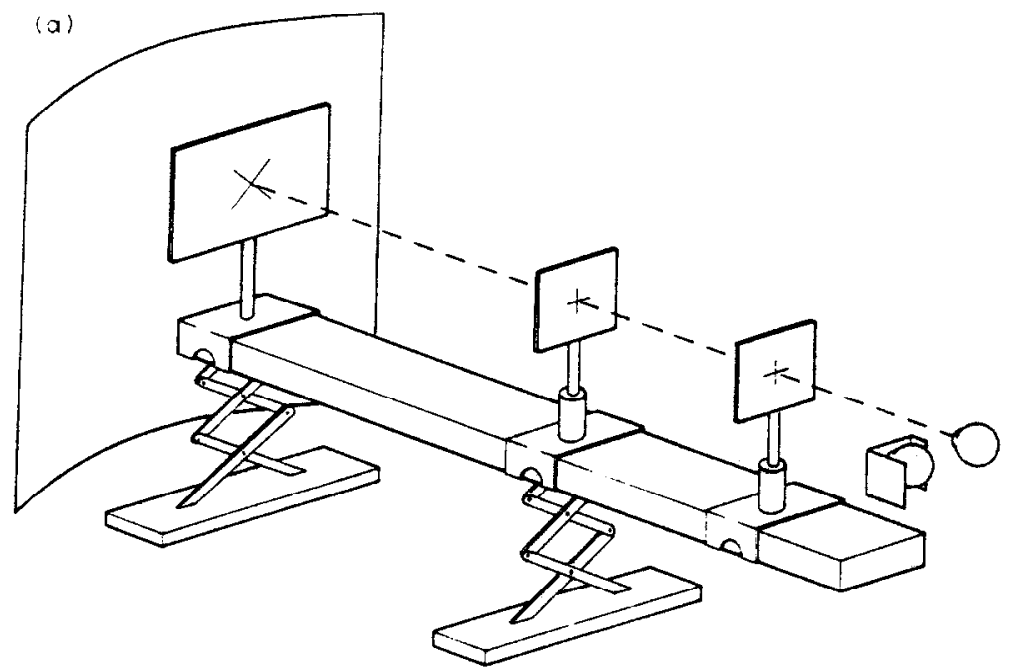

Translucent background screen

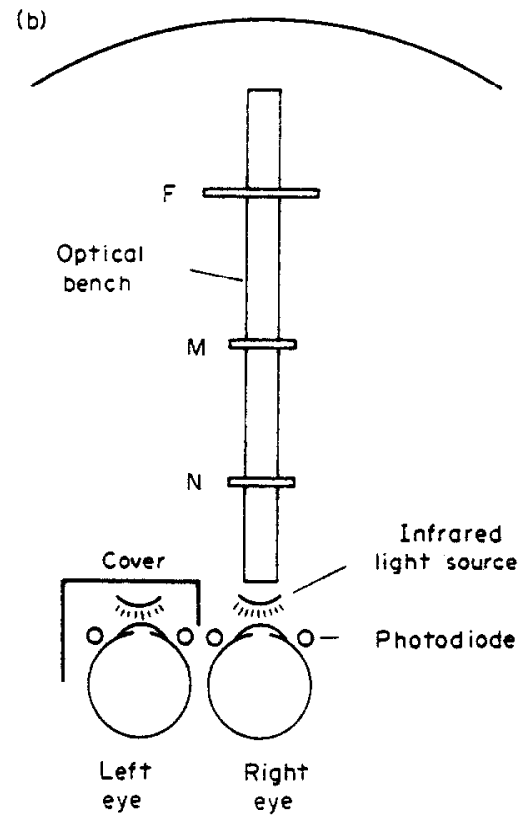

Fig. 1. Schematic representation of apparatus and eye alignment. Three targets on an optical bench were aligned along the iitw of sight of the viewing eye at distances of 100,50 and $25 \mathrm{~cm}$. They were viewed against a large, dim, homogeneous background screen (a). The fellow eye was prevented from seeing the targets and the surrounding objects by interposing an occluder and drape (cover). Photodiodes and infrared light sources were positioned close to the eyes (b).

middle and near targets, respectively. The far target consisted of a fine white " $X$ " painted upon a large, black matte background; the " $\mathrm{X}$ " subtended a visual angle of $2.5^{\circ}$, while the white lines forming the " $X$ " subtended a visual angle of $7 \mathrm{~min}$ arc. The luminance of the middle and near targets was $0.5 \log \mathrm{ft}-\mathrm{L}$; for the far target, it was -0.6 $\log \mathrm{ft}-\mathrm{L}$. The experimenter individually illuminated the targets with a silent, three-pole rotary switch.

For purposes of alignment, the targets were simultaneously illuminated and then adjusted by the subject until the target intersections were superimposed. Since the etched vertical line of the near target subtended a visual angle of $4 \mathrm{~min}$ arc, the alignment of the targets was accu- rate to less than $\pm 2 \mathrm{~min}$ arc. To determine the effect of target misalignment on the eye movements, the far end of the optical bench was rotated $0.5 \mathrm{~cm}$ to either side of the subjective target alignment position, and an "offset" run was performed at the end of an experimental session.

\section{Occlusion technique and eye measurement}

Throughout the experiment the viewing eye could readily see the targets. However, except for the initial and final calibrations, the fellow eye was oceluded by a large, black, matte-surfaced partition which blocked its view of the targets. In addition to the partition, a black felt cloth was draped over the partition and the side of the subject's 
head to completely block any peripheral view of objects in the laboratory. This occlusion technique allowed eye movements to be recorded from the covered eye. The room was darkened to reduce peripheral distractions to the viewing eye. and a large. translucent screen was placed behind the far target to produce a dim. homogeneous background. The subject's head was stabilized by a headrest and a bite bar covered with dental impression material.

The horizontal position of each eye was measured by the photoelectric technique (Stark. Vossius and Young. 1963). A small. battery-powered penlight with an infrared filter (Kodak Wratten No. 88) placed in front of the light source diffusely illuminated each eye. Photodiodes (Texas Instruments. LS 400) were positioned perpendicular to the nasal and temporal limbal regions of each eye. They were placed $7 \mathrm{~mm}$ from the covered eye, were linear over a range of 14 degrees, and had a noise level of $6 \mathrm{~min}$ arc; they were placed $3 \mathrm{~mm}$ from the viewing eye, were linear over a range of $2.5^{\circ}$, and had a noise level of $2 \mathrm{~min}$ arc. The signals from the sensor pair of each eye were differentially amplified and recorded, along with the stimulus, with an FM instrumentation tape recorder (Ampex FR1300). The band width of this recorded data was $1000 \mathrm{~Hz}$.

\section{Target sequencing in experimental runs}

The targets were presented in either a non-predictable or a predictable manner. For the non-predictable portion of an experimental run, the middle target was initially illuminated. Subsequent target illumination could be. at random times. to either the near or the far target with simultaneous extinction of the middle target illumination. Thus, both temporal and spatial randomization of target presentations was achieved. Following a variable pause. the target illumination was then changed back to the middle target, thereby producing temporal randomization only. The subjects were presented a series of these cycles and instructed to maintain clarity of the illuminated target. During the predictable portion of an experimental run, only the middle and near targets were utilized. Each target was alternately illuminated for approximately equal durations, thus enabling the subject to predict both the time and direction of the next presentation.

An experimental session began with a calibration of the right and the left eyes under binocular conditions. Following occlusion of an eye, the calibration procedure was repeated. The targets were presented to the subject in blocks of non-predictable, predictable, and non-predictable trials, in sequence, with calibration checks between each block of trials. An offset run followed, and a final calibration determination under both monocular and binocular viewing conditions ended the session.

\section{Subjects}

Five graduate students, one being a näve observer, served as subjects. The targets were aligned along the line of sight of the dominant eye, as determined by the sighting method, in all five subjects; the non-dominant eye was also tested in two of these subjects. The middle and near targets were placed well within the subjects' far and near points of clear vision without need of corrective lenses. Consequently, only data for eye movements between these two targets were incorporated into our analysis. No subject had residual astigmatism of greater than 0.37 diopters or subnotmal visual acuity.

\section{Data aniysis}

Fot analysis, the data were subsequently played back into either a digital computer (Digital Equipment Corp. PDP-8/1) or a four-channel strip chart recorder (Sanbom No. 150). The bandwidths of the digitized data and the chart recorder were 500 and $70 \mathrm{~Hz}$, respectively.

All of the vergence responses recorded for each of out subjects at each session were incorporated into our fre- quency analysis. For our amplitude analysis. 131 out of 345 total responses were used in the averages for the dominant eye viewing condition; 45 out of 70 total responses were utilized in the averages for the non-dominant eye viewing condition.

\section{RESULTS}

\section{Binocular movements}

During accommodative vergence, the initial response was a disjunctive, generally synchronous $190 \%$ of the time), movement in the two eyes as clearly displayed in Figs 2-7 and 9-11. Thus, contrary to previous reports describing human accommodative vergence as a uniocular phenomenon (Alpern and Ellen, 1956; Hermann and Samson, 1967), we found the accommodative vergence response to be binocular in nature, as is true for other types of vergence. We studied, in detail, approximately 415 movements in our five subjects viewing with either the dominant or non-dominant eye, and all exhibited this binocular response. These binocular movements agreed in direction, but not in amplitude, with movements predicted by Hering's law of equal innervation. In Figs 2-7, movement in both the covered eye (upper trace) and the viewing dominant eye (middle and lower traces) is evident, especially when the record of the viewing eye's movement is expanded (lower trace). This expanded trace in our triple displays allows easier observation and analysis of the movement characteristics of the viewing eye, while comparison of upper and middle traces, whose calibrations are approximately equal, dramatically demonstrates the differences in amplitudes of the vergence movements in each eye.

\section{Vergence attenuation}

The average magnitude of the total vergence movement (excluding the saccadic components) for 131 movements in four subjects in the dominant viewing eye was $0.41^{\circ} \pm 0.22^{\circ}$ (s.d. of an observation) with a range from 0.08 to $1.5^{\circ}$; the average magnitude of the total vergence movement in the covered eye was $3.5^{\circ} \pm 0.91^{\circ}$ (s.d. of an observation) with a range from 2.0 to $7.0^{\circ}$. Thus the vergence movement in the viewing eye was attenuated in all our subjects, on the average, approximately $88 \%$ with respect to the movement in the covered eye.

\section{Types of movements}

During our analysis of the eye movement records, several characteristic patterns of movement were observed in the dominant viewing eye in response to the accommodative input. This led us to classify these movements (Fig. 8) and to establish frequency distributions for our subjects' responses (Table 1).

When no discernible movement that appeared either correlated with the onset of the stimulus or to the movemient in the covered eye was present, it was classified as a Type 0 response. This response was not observed in any of the movements of our subjects.

The Type I response, displayed in Figs. $2 a, b, 4 a$ and 8 , began with a vergence movement that carried the eye away from the target. About $150-350 \mathrm{msec}$ later, a smooth movement returned the eye to the 


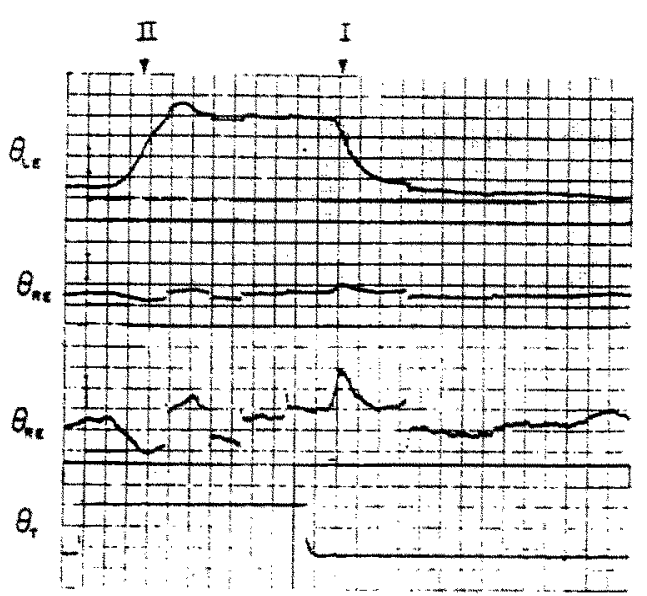

(o)

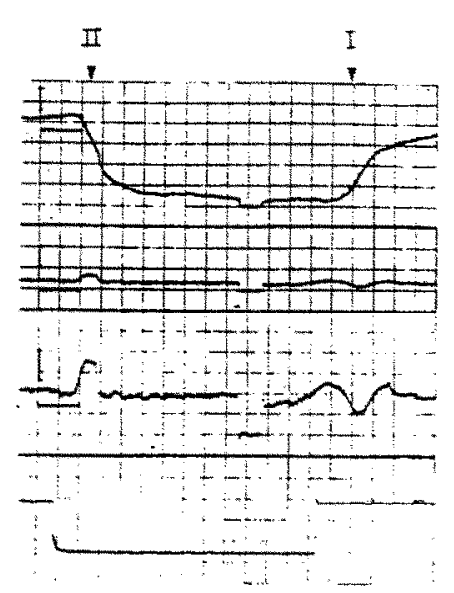

(b)

Fig. 2. Binocular eye movements for subject R.V.K. Shown from top to bottom as a function of time are covered left eye position, viewing right eye position (gain approximately equal to left eye). viewing right eye position (gain greater than left eye), and stimulus (onset of middle target denoted by upward deflections and onset of near target denoted by downward deflections). respectively. Calibration bars represent $0.25^{\circ}$ for viewing eye (lower trace), $1.0^{\circ}$ for the covered eye and viewing eye (upper and middle traces) and 400 milliseconds. Leftward movements represented by upward deflections. This convention will be used in Figs 2-7 unless otherwise noted. Convergence and divergence movements are shown for nonpredictabie (a) and predictable (b) stimulus presentations. In (a), note Type II movement during divergence. microsaccades. and then Type 1 movement during convergence. In (b), note Type II movement during convergence, single large microsaccade. and then Type I movement.

initial baseline position. It should be noted that during this smooth return manoeuver, both eves moved in the same directions. The amplitude of the total vergence movement in the viewing eye was $0.22^{\circ} \pm 0.11^{\circ}$ (s.d. of an observation) and $5.1^{\circ} \pm 2.1^{\circ}$ (s.d. of an observation) in the covered eye. This Type I movement, present in four of our subjects. was an infrequent response $(4 \%)$, occurred primarily during the nonpredictable stimulus presentations $(98 \%$ of the time), tended to occur more frequently during convergence, and accounted for the greatest average vergence attenuation in the viewing eye $(96 \%)$.
In the Type II response, shown in Figs. 2a, b, 3a, b, $4 \mathrm{~b}, 8,9 \mathrm{~b}$ and $10 \mathrm{~b}, \mathrm{c}$, approximately $160 \mathrm{msec}$ following the initiation of the vergence movement, an abrupt change in eye velocity was apparent. This was followed by a saccade $120-400 \mathrm{msec}$ later that returned the eye to the target. Sometimes this late saccade had a large, dynamic overshoot (Bahill, Clark and Stark, 1975), while only infrequently was static overshooting observed. The total vergence amplitude in the viewing eye was $0.29^{\circ} \pm 0.08^{\circ}$ (s.d. of an observation) and $3.7^{\circ} \pm 0.9^{\circ}$ (s.d. of an observation) in the covered eye. This Type II response, observed in four

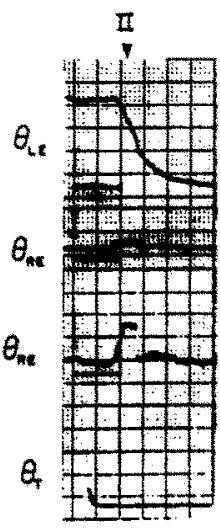

(o)

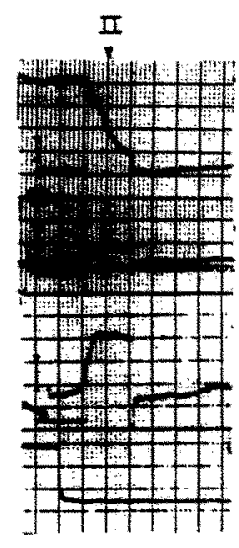

(b)

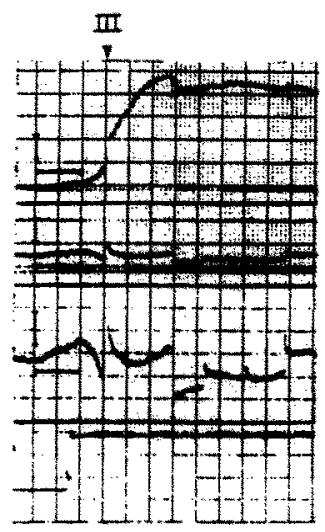

(c)

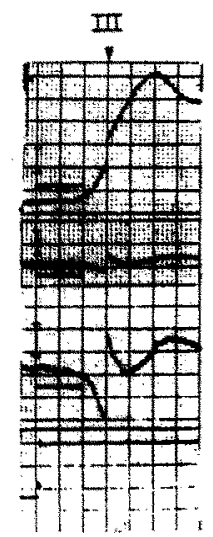

(d)

Fig. 3. Binocular eye movements of subject R.V.K. for nonpredictable $(a, d)$ and predictable $(b, c)$ stimulus presentations. Type II responses $(a, b)$ show variable duration of "fat-tops". Type III responses (c. d) show large dynamic overshoots in saccadic movements; overshoots also present in fixation microsaceades. 


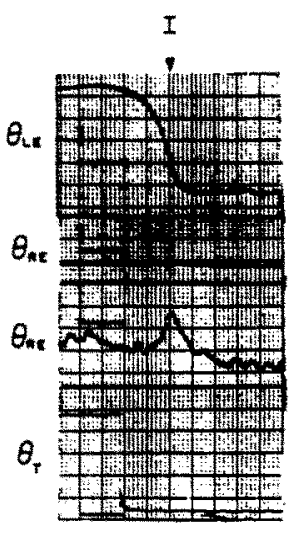

(a)

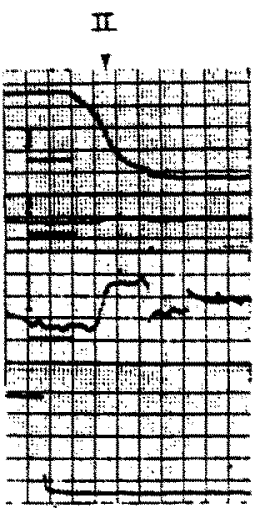

(b)

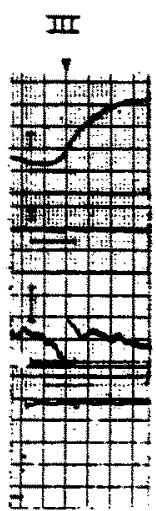

(c)

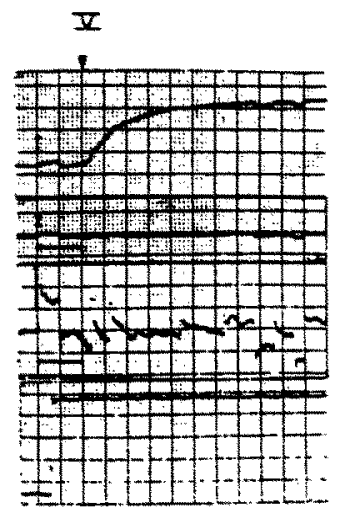

(d)

Fig. 4. Binocular eye movement for subject KJ.C. Shown from left to tight are Type I, Type II, Type III, and Type V movements. Type I movement shows marked asynchrony (up to $400 \mathrm{~ms}$ ) for beginning of vergence response between two eyes. The Type $V$ response appears to be repetitive Type III movement: note the conjugate microsaccade preceding vergence response. Abrupt changes in eye velocity of viewing eye produced "flat-top" regions characteristic of Type II response. Stimuli were predictable for the Type II and V responses.

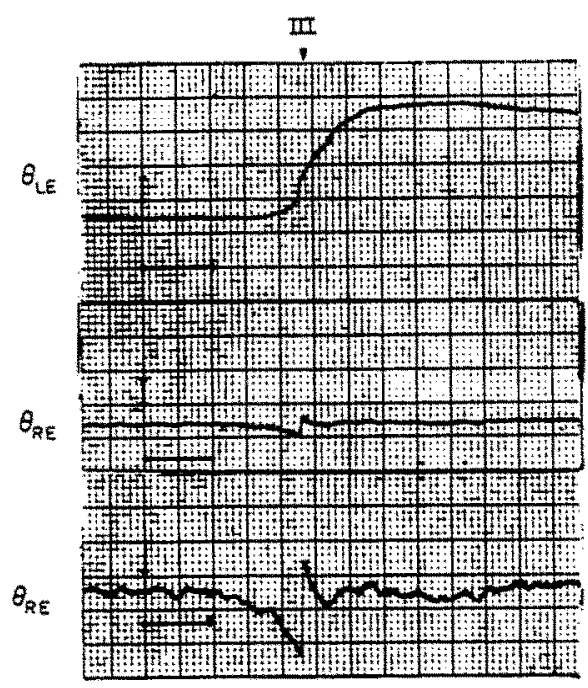

(a)
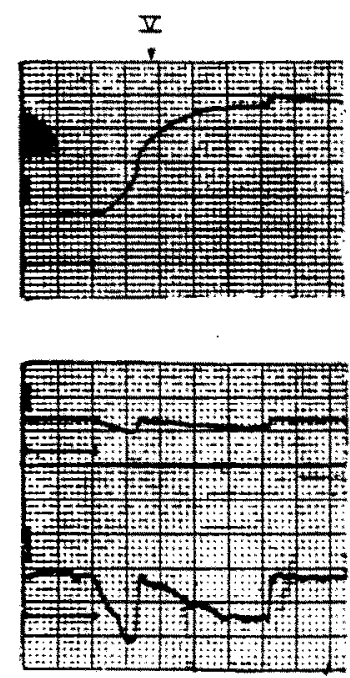

(b)

Fig. 5. Binocular eye movements of naive subject C.F.F. during divergence to nonpredictable stimulus presentations. Shown are Type III movement (a) and miscellaneous Type V movement (b) which appears to be compound variety (Type III/I). Stimulus trace not shown.

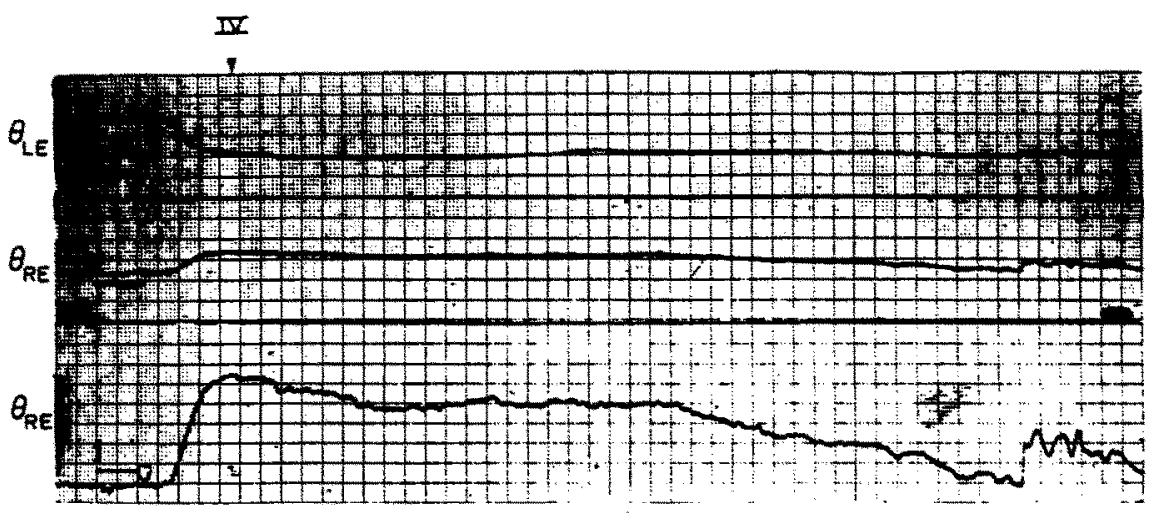

(a)

Fig. 6. Binocular eye movement of subject C.F.F. during convergence to nonpredictable stimulus presentation. Shown is Type IV movement observed in $33 \%$ of recorded movements for this subject. Total response time is about 9 secs. Stimulus trace not shown. 


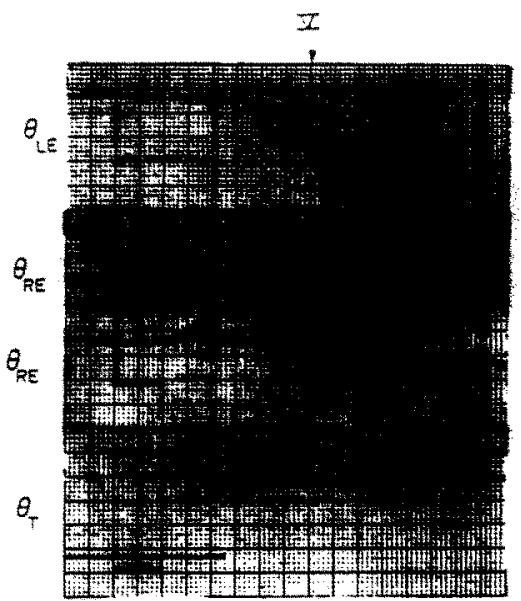

(a)

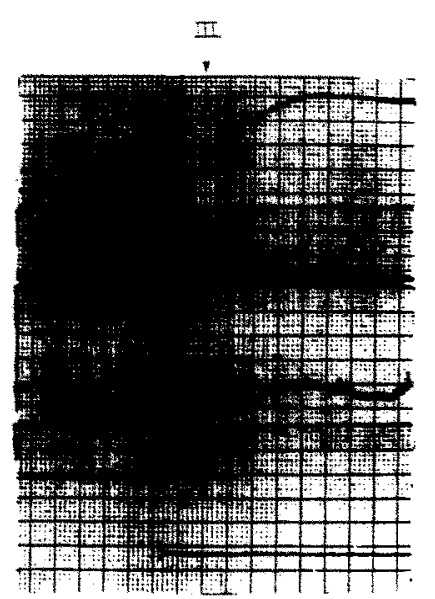

(D)

Fig. 7. Binocular eye movements of subject S.W. for nonpredictable stimulus presentations. Upward deflections of stimulus marker denote onset of near target, and downward deflections denote onset of middle target. Trace (a) shows Type $\mathrm{V}$ movement which appears to be overlapping succession of Type III movements: trace (b) shows Type 111 movement with small intial vergence component.

of our subjects, occurred with moderate frequency $(17 \%)$ and was uninfluenced by mode of presentation but tended to occur more frequently during convergence in some subjects, and resulted in a large a verage vergence attenuation in the viewing eye $(92 \%)$.

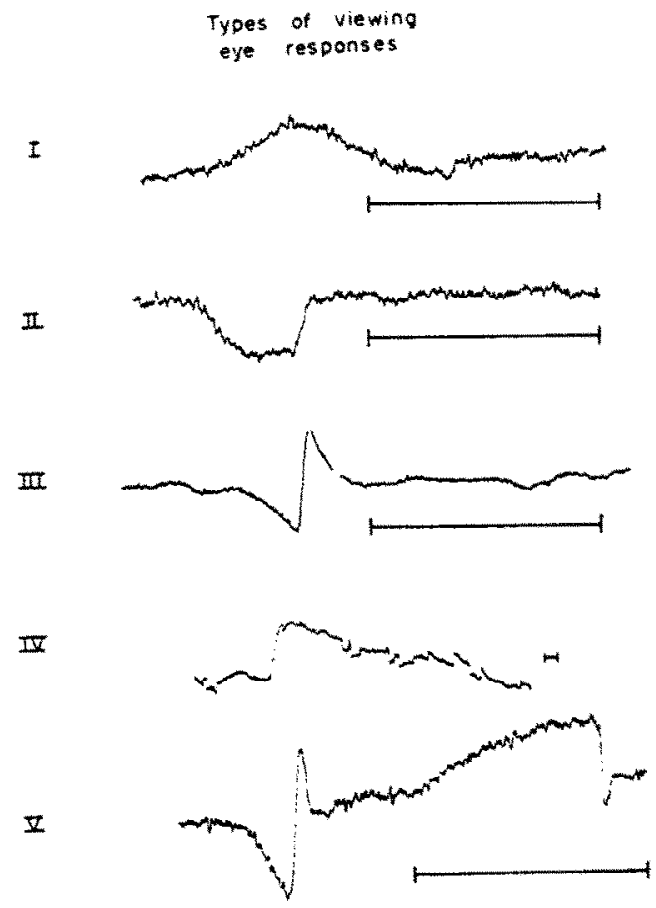

Fig. 8. Basic types of eye movement and their frequency observed in dominant viewing eye (Types I-V. from top to bottom). In Type I $(3.76 \%$, vergence corrected by smooth movement. In Type II (16.81\%) vergence corrected by late saccade, while in Type III $(37.68 \%)$ vergence inter. rupted by early pre-programmed saccade. In Type IV (11.59\%), vergence present without early correction; in Type V $(29.85 \%)$ are miscellaneous responses. Type 0 movement never observed in subjects' responses. Latencies averaged $200 \mathrm{~ms}$. Calibration bars represent $500 \mathrm{msec}$.
The distinguishing feature of the Type III movement shown in Figs. $3 c, d, 4 c, 5 a .7 b, 8,10 a$ and $11 \mathrm{a} . \mathrm{b}$ was an early saccade, occurring approximately $160 \mathrm{msec}$ after the start of the vergence, that was superimposed upon the vergence movement. This sac. cade, which usually had a large dynamic overshoot (Bahill, Clark, and Stark, 1975), drove the eye back beyond the target position, and the continuing vergence movement placed the eye directly in line with the target (as inferred by the lack of saccadic corrections subsequent to the vergence completion). The amplitude of the total vergence movernent in the viewing eye was $0.47^{\circ} \pm 0.24^{\circ}$ (s.d. of an observation) and $3.3^{\circ} \pm 0.63^{\circ}$ (s.d. of an observation) in the covered eye. This Type III movement, present in all of our subjects, occurred with the greatest frequency $(38 \%)$, tended to be uninfluenced by the mode of stimulus presentation, was principally associated with divergence movements $(85 \%$ of the time), and accounted for the smaller average vergence attenuation $(86 \%)$. However, the vergence attenuation appeared to be related to the subject's experience in eye movement experiments. Those subjects with the most experience exhibited the greater average vergence attenuation $(88 \%)$, while subject C.F.F. with the least experience manifested the smaller average vergence attenuation $(71 \%)$, although no conscious attempt was made by any of the subjects to influence the motor response to the sensory input by introducing learned ocular "gymnastics" or tricks" during the experiment.

One of the most unusual responses was the Type IV, shown in Figs 6 and 8 , in which a large vergence movement drove the eye off the target and a slow movement (total response time about $9 \mathrm{sec}$ ) returned the eye back to the target. During our experimental sessions, this Type IV movement was observed with noteworthy frequency only in subject C.F.F. $136 \%$ of his responses) and accounted for $12 \%$ of the group response. The average amplitude of the vergence movement in the viewing eye was $0.6^{\circ} \pm 0.13^{\circ}$ (s.d. 
Table 1. Frequency and percentage of categorized eye movements in the dominant viewing eye for nonpredictable (NP) and predictable (P) stimulus presentations

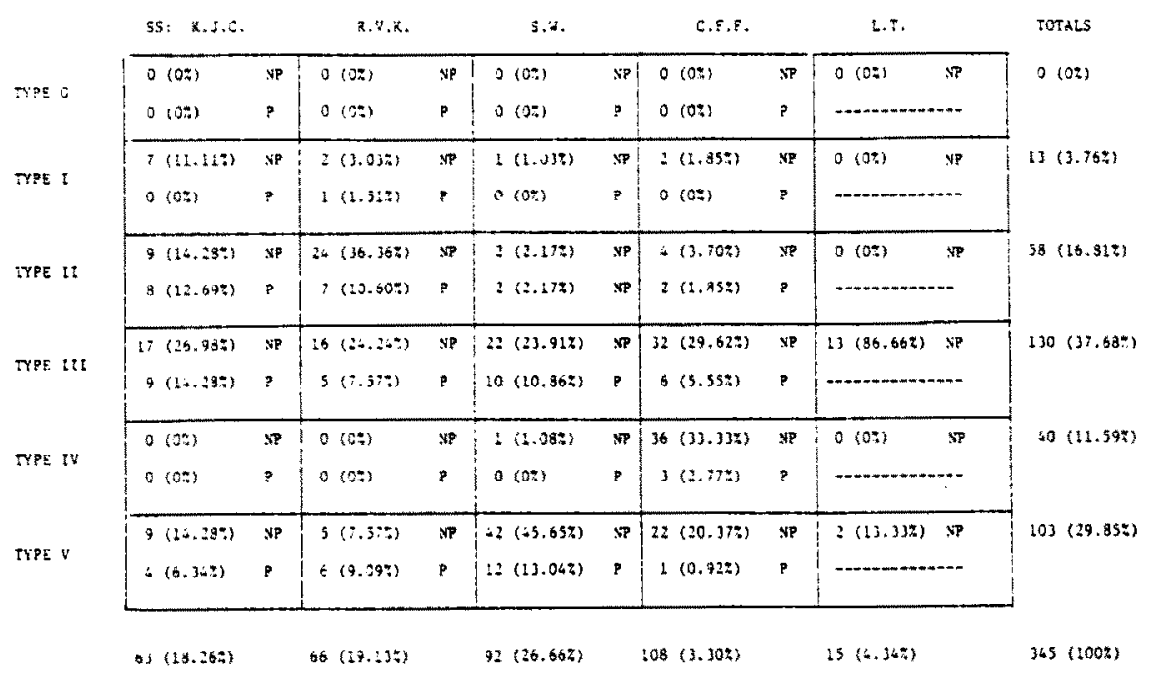

of an observation) and $3.3^{\circ} \pm 0.6^{\circ}$ (s.d. of an observation) in the covered eye. This movement primarily occurred in the nonpredictable mode, was only observed during convergence movements. and resulted in moderate vergence attenuation in the viewing eye $(82 \%)$. The underlying mechanism producing this movement remains elusive.

Into the last category, Type V, were placed our miscellaneous responses, shown in Figs $4 d, 5 b, 7 a$, 8 and 9a. Many of these movements appeared to be combinations of Types I-III and consisted of either a mixed sequence of the three types or a succession of one type of movement. For example. the multiple saccades shown in Fig. 7a appear to be composed of overlapping Type III movements. A few responses did not fit into any particular I-IV category. Also placed in to this Type $V$ category were those movements in which blinks or other sources of artifact partially obscured either the initial or final baseline eye position without interfering with the essential response; these types of responses could still be categorized and were used for our frequency analysis only. This type $\mathrm{V}$ response, present in all of the subjects. occurred with moderate frequency $(30 \%)$. tended to be found more frequently during predictable presentations in some subjects, and was not related to vergence direction. Vergence amplitudes and vergence attenuations were appropriate for the type of movements executed.

\section{Intra- and inter-subject variability}

An interesting facet of this investigation was the intra- and intersubject variability with respect to the frequency of the movements present on a given day of testing. The same subject (K.J.C.), tested on two separate occasions, executed Type II and Type III movements with equal frequency on one day, but virtually all Type III movements on a second day. Subject R.V.K.. tested on three separate days, made ap- proximately an equal number of Type II and Type III movements at each session. Regarding intersubject variability, subjects S.W. and.L.T. executed primarily Type III and Type $V$ movements, while C.F.F. made Type III and Type IV movements with relatively equal frequency.

Predictable us nonpredictable stimulus presentation modes

The effect of target presentation mode, that is, predictable and nonpredictable stimulus sequencing; on accommodative vergence responses was investigated with our experimental paradigm; the results in terms of types of viewing eye movement were considered in the previous sections. There were no marked trends in the responses as a function of stimulus presentation mode. This is readily apparent from Table 2 which presents the average values of the categorized eye movements for four subjects under both stimulus conditions. For the nonpredictable mode, the average magnitude of the total vergence in the viewing eye was $0.41^{\circ} \pm 0.21^{\circ}$ (s.d. of an observation) and $3.50^{\circ} \pm 0.97^{\circ}$ (s.d. of an observation, $n=95$ ) in the covered eye; for the predictable mode, the average magnitude of the total vergence in the viewing eye was $0.42^{\circ} \pm 0.22^{\circ}$ (s.d. of an observation) and $3.59^{\circ} \pm 0.75^{\circ}$ (s.d. of an observation, $n=36$ ) in the covered eye. Only the covered eye values for subjects S.W. and K.J.C. (Type III) appear to show large (almost one degree) differences.

\section{Effects of eye dominance}

Several interesting results were obtained when the nondominant eye was used (Figs 9, 10). Two subjects, K.J.C. and R.V.K., were tested with their nondominant, left eye as the viewing eye during a predictable experimental run. For both subjects, the average amplitude of the total vergence movement (Types 1-III) was $0.47^{\circ} \pm 0.14^{\circ}$ (s.d. of an observation) with 
Table 2. Vergence amplitudes $\left(\bar{X}^{2} \pm\right.$ I S.D.) for predictable (P) and non-predictable (NP) presentations in covered eye (CE) and dominant viewing eye (VE)

\begin{tabular}{|c|c|c|c|c|c|c|c|c|c|}
\hline & is: & $3 . \because x$ & & $x, 2.2$. & & \multicolumn{2}{|l|}{ s.t. } & \multicolumn{2}{|l|}{$\therefore \therefore}$, \\
\hline & $\approx$ & 2.55 & $=1$ & $4.36=0.32$ & $n=\dot{4}$ & & & \multirow{2}{*}{\multicolumn{2}{|c|}{ 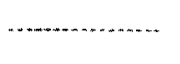 }} \\
\hline & VE & $2: 2$ & $\mathrm{x} F$ & $2.2 *=0.40$ & $i ; ?$ & \multicolumn{2}{|c|}{ 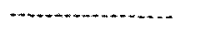 } & & \\
\hline \multirow{2}{*}{ rint 1} & 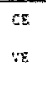 & $\begin{array}{l}2.90 \\
0.22\end{array}$ & $\begin{array}{l}: 1 \\
p\end{array}$ & (n) & & - & -.. & \multicolumn{2}{|c|}{ - } \\
\hline & CE & $3.16=0.31$ & $n=23$ & $4.38=0.91$ & $\pi=?$ & & & & \\
\hline \multirow[t]{5}{*}{ TY? 11} & VE & $0.31=0.09$ & P & $0.27=0.98$ & $y q$ & & & & \\
\hline & & $3.14: 0.53$ & $n=\hat{s}$ & $4.54=0.49$ & $n=7$ & \multirow{2}{*}{\multicolumn{2}{|c|}{ (4)- }} & \multirow{2}{*}{\multicolumn{2}{|c|}{ 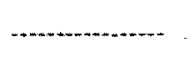 }} \\
\hline & 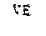 & $0.33=0.07$ & $p$ & $0.24=0.07$ & $?$ & & & & \\
\hline & $c \Sigma$ & $3.22=0.57$ & $n=10$ & $\therefore .03=0.36$ & $n=14$ & $3.02=0.29$ & $n=13$ & $2.8 t=0.39$ & $\because x$ \\
\hline & ve & $0.22=0.16$ &. $\mathrm{PP}$ & $0.32=0.62$ & NP & $0.39=0.08$ & NP & $2.89=3.26$ & $s z$ \\
\hline \multirow{3}{*}{ Type :t! } & $c z$ & $3.32: 0.21$ & $n=4$ & $3.09=0.71$ & $n=5$ & $3.7 \%=0.69$ & $n=7$ & $3.03=0.34$ & $\because \cdots$ \\
\hline & $\mathrm{vE}$ & $0.59=0.16$ & 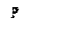 & $0.34=0.12$ & $?$ & $0.39=0.07$ & 8 & $\alpha+2=4.13$ & $?$ \\
\hline & $\mathrm{CE}$ & 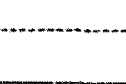 & & & & $n \in m$ & & $\begin{array}{l}3.24=2.35 \\
0.39=0.23\end{array}$ & $=$ \\
\hline TYPE :Y & $\begin{array}{l}\mathrm{CE} \\
\mathrm{rE}\end{array}$ & - & & 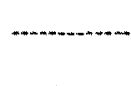 & $-\rightarrow$ & $\cdots$ & & $\begin{array}{l}3.58=0.30 \\
0.50=0.17\end{array}$ & $=$ \\
\hline
\end{tabular}

the nondominant viewing eye, but only $0.32^{\circ} \pm 0.09^{\circ}$ (s.d. of an observation) when the dominant eye was used. For subject K.J.C. (Fig. 9), while the movements occurred in the same categories as during the predictable run with the dominant viewing eye, fewer Type II responses were present. Moreover, the vergence attenuations were smaller. For example, in the Type II and Type III responses, the vergence reductions averaged $93 \%$ when the dominant eye was the viewing eye, but only $73 \%$ with the nondominant eye. Also, a characteristic double vergence response (Fig 9a) occurred with high frequency $(45 \%$ of the time) that was never observed in the dominant eye responses: furthermore, the vergence attenuation for this response was only $58.1 \%$. For R.V.K. (Fig. 10), the movements occurred in the same categories, had similar frequencies, and exhibited similar vergence attenuations as during the predictable run with the

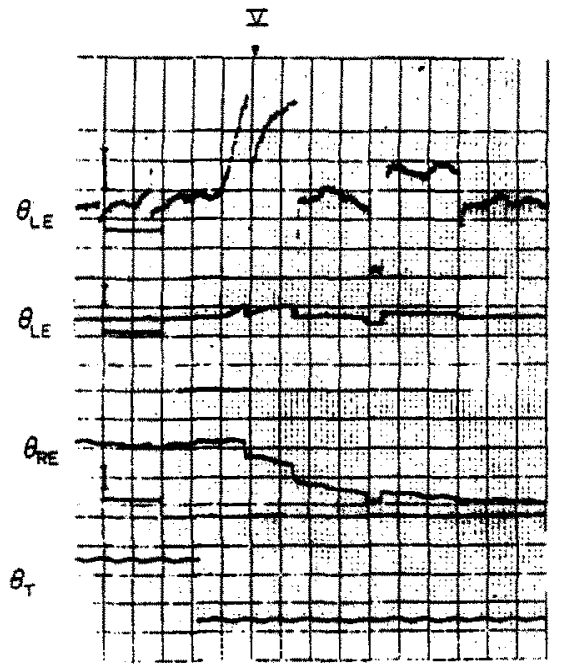

(a)

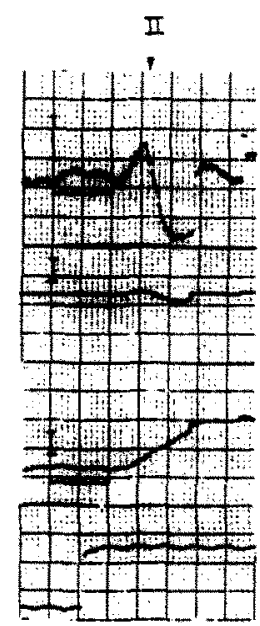

(b)

Fig. 9. Binocular eye movement responses of subject K.J.C. when non-dominant left eye viewed target during predictable run. Shown are left eye position (high gain), left eye position (low gain), right eye position (gain approximately equal to low gain of left eye), and stimulus fupward deflections denote onset of near target, and downward deflections denote onset of middle target), tespectively, from top to bottom as function of time. Calibrations same as other figures. Note unusual "double vergence" Type $V$ response (a) that was never observed when the dominant eye viewed target and early smooth component (b) prior to Type II response. 


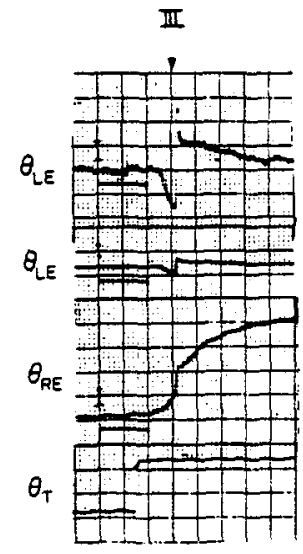

(a)

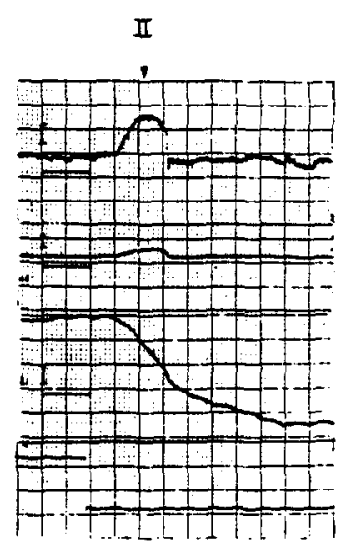

(b)

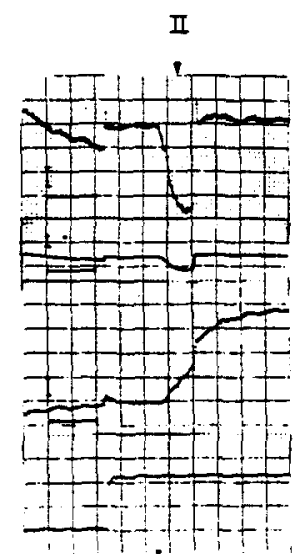

(c)

Fig. 10. Binocular eye movements for subject R.V.K. when non-dominant left eye viewed target during predictable run. Conventions same as Fig. 9. Note typical III response (a) and pronounced smooth movements of viewing eye in Type II response (b, c) which began to return eye toward baseline position prior to late error-correcting saccade.

dominant viewing eye. However, with the dominant eye, Type III movements were generally present $(85 \%$ of the time) during divergence, while with the nondominant eye Type III movements occurred only during convergence.

\section{Saccadic attenuation}

Similar to the vergence attenuation noted in the viewing eye during accommodative vergence, attenuation of some saccadic movement, mainly in the Type
III response, was noted. After allowing for compensations for the ongoing vergence in each eye, the amplitudes of the saccades in the viewing eye were as much as $20 \%$ smaller than those in the covered eye.

\section{Offset experiment}

Eye movements during the offset experiment exhibited an expected shift in baseline eye position superimposed on the typical Type I-V accommodative vergence responses. The lack of any baseline shift during

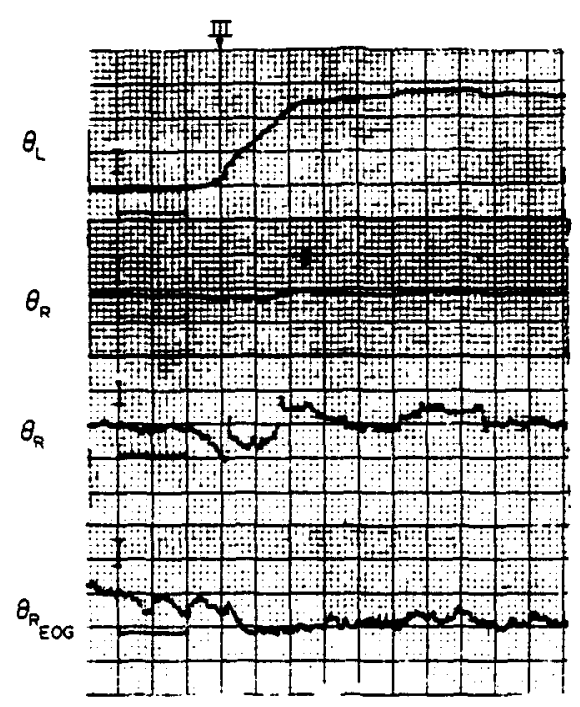

(a)
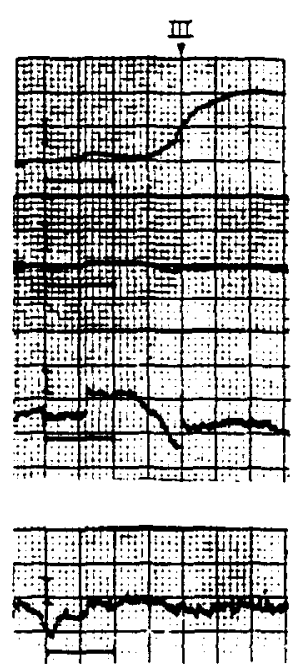

(b)

Fig. 11. Binocular eye movements of subject K.J.C. with the dominant right eye viewing target during divergence. Viewing eye monitored simultaneously with photoelectric and electro-oculographic methods; covered eye monitored photoelectrically. Shown are covered left eye position, viewing right eye position (gain approximately equal to left eye), viewing right eye position (gain greater than left eye), and viewing eye position (electro-oculographic method). respectively, from top to bottom as a function of time. Calibrations same as other figures. Note clearly observable Type III movement in viewing eye monitored with photoelectric technique, but only noise and drift in viewing eye during same time course with electro-oculographic recording, thus demonstrating lack of correlation in detected movement between these two techniques for small ocular rotations. 
the an-axis accommodative vergence conditions reinforces the fact that the targets were precisely aligned.

\section{DISCUSSION}

During accommodative vergence, binocular vergence movements were observed in all of the several hundred eye movements analyzed in our five subjects: this included viewing with the dominant and nondominant eye, predictable and nonpredictable stimulus presentations, and for convergence and divergence. Since these records clearly demonstrate the binocular nature of accommodative vergence, why has this phenomenon remained unnoticed? The movements in the viewing eye were rarely greater than one degree in amplitude, and, in fact, averaged $0.41^{\circ} \pm 0.22^{\circ}$ (s.d. of an observation) in amplitude. The inherent noise level of the electro-oculographic recording method used by Alpern and Ellen (1956) could easily mask these small eye movements. Moreover, electro-oculographic signals are usually filtered at $10-25 \mathrm{~Hz}$ and such low-pass filtering, which is helpful in reducing this noise, will distort these small movements. Figure 11 clearly shows the differences in the eye movement traces during accommodative vergence when the photoelectric and electro-oculographic methods were utilized simultaneously. The photoelectric technique, which Hermann and Samson (1967) used to measure eye movements during accommodative vergence, will also fail to register some of these small movements if the experiment is not specifically designed to detect very small ocular rotations. For example, when the photo-cells are positioned far from the eyes, a strategy used to increase the linear operating range of the system, resolution capabilities will decrease. Reduction in sensitivity with the photoelectric method is also encountered when low-pass filtering of $50 \mathrm{~Hz}$ or less is introduced.

Hindsight allows us to look back at the published eye movement recordings of the previous investigators with sharper focus. We feel that the data of Alpern and Ellen (1956) demonstrate a baseline change in the viewing eye during accommodative convergence; this initial baseline shift is consistent with a small, leftward movement (convergence) in the viewing right eye (their Fig. 2) as the covered left eye rotates rightward. The data of Hermann and Samson (1967) clearly shows vergence and saccadic movements taking place in the viewing eye during accommodative vergence. Thus, due to either state of the art technical limitations at the time of the previous researches, failure to design the experiment with detection of fine ocular rotations as a primary investigative goal, or not having analyzed the data in a detailed manner, these small movements in the viewing eye associated with accommodative vergence have rec.ived little attention, and therefore have resulted in obscuring its true binocular nature.

These findings regarding binocularity help to place accommodative vergence in a stricter accordance with Hering's law of equal innervation to corresponding eye muscles, certainly a basic tenet of oculomotor physiology. Our results show that the two eyes do move in the appropriate directions, but by unequal amounts, durng accommodative vergence However. due to the stimulus conditions imposed on the visual system during this experiment, the binocular oculomotor response still, in part, violates Hering's law: but it does so to a lesser degree. What might start as an equal, two-eyed response is modified by multilevel control processes into an unequal. but still binocular response, in the interest of maintaining the viewing eye on the target. Thus, accommodative vergence can now be grouped with other binocular vergences, and not thought of as some enigmatic, uniocular phenomenon.

By analysis of the spatial and temporal characteristics of the movements in the viewing eye, one can begin to understand the underlying levels of neural control that might be operating during accommodative vergence. In the Type I response. the smooth movement corrected the error produced by the initial vergence. This suggests that the smooth movement is under visual feedback control and simply functions to correct retinal position and/or velocity error.

The Type II responses contained both position and velocity correcting components. In response to a target change, the vergence movement smoothly drove the viewing eye away from the target, and thus created a velocity error on the retina between the eye and the target which, in turn. stimulated the smooth pursuit system to reduce this velocity error. We feel that the "flat-top" regions in the viewing eye's response represents cancellation of the vergence by the opposing smooth movement. Sometimes the smooth movement actually reversed the direction of movement in the viewing eye for a short period (Fig. $10 \mathrm{~b})$; at these times, the smooth movement velocity was probably greater than the vergence velocity. Evidence for early introduction of a smooth mechanism is shown in Fig. 9b where a smooth movement occurs prior to the initial vergence movement. In this figure, the smooth movement has shorter latency and $/$ r greater velocity than the vergence component. If the latency and the velocity of the opposing smooth and vergence movements were similar, the viewing eye would reamin steady for the duration that the two movements were matched. The asynchronous vergence movements shown in Figs $4 a, b$, where the viewing eye remains steady for up to $400 \mathrm{msec}$ during the initial vergence phase, can be explained on this basis. Thus, a smooth compensatory mechanism appears to be one strategy adopted by the oculomotor system to minimize the excursions in the viewing eye. The early smooth movement probably represents a preprogrammed strategy, while the later smooth movement is under visual feedback control. The interaction of smooth pursuit and accommodative vergence has been hypothesized by Keller (1973). The saccade of the Type II movement, due to its generally late occurrence in the movement, and its amplitude being approximately equal to the position error introduced by the vergence movement. leads us to believe that it simply attempts to correct the residual retinal error and represents visual feedback control.

However, in the Type III movement, since the saccade occurred so early in the movement, and its amplitude was always larger than the error in eye position at the time of the estimated saccadic sampling period. we feel that this movement represents 
a high-level preprogrammed control strategy to maintain the target within the foveal region and does not represent a simple position and velocity error-correcting mechanism as in the Type II responses.

It is interesting to consider the size of these viewing eye movements in relation to the diameter of the sensitive foveal region ( $\sim 40 \mathrm{~min}$ arc). The average total vergence in the Type I and II movements was 0.22 and $0.29^{\circ}$, respectively, while in the Type III movement, the average total vergence was $0.47^{\circ}$. In the Type I and II movements the eye moved until the target was positioned near one edge of the foveal zone. In the Type I response. the smooth movement reversed the eye's direction to once again place the centre of the fovea coincident with the target. In the Type II response, the smooth movement cancelled the vergence movement and maintained the target slightly eccentric to the foveal center (while the covered eye continued its vergence movement) until the pusition error in the viewing eye was corrected, through visual feedback control, by a saccade. However, the Type III strategy was quite different. The initial vergence movement $\left(0.27^{\circ} \pm 0.14^{\circ}\right.$, s.d. of an observation) brought the target to one edge of the fovea, the saccade moved the eye so that the target was now at the other edge of the fovea, and the continuing vergence placed the fovea coincident with the target. Smooth pursuit-vergence interaction was evident in the last portion of this movement; once the continuing vergence centered the target on the fovea, the viewing eye remained stationary as vergence in the covered eye ensued. The Type III movement made use of the total foveal zone, thus producing more total vergence than Types I or II, while still keeping the target within the foveal region.

This quantitative analysis of the viewing eye movements during accommodative vergence suggests an overall multi-strategy fixation-holding mechanism; such strategies include preprogrammed saccadic and smooth movements, saccadic attenuation, and smooth and saccadic movements under visual feedback control. The aim of this mechanism is to maintain the target within this sensitive, central foveal zone during accommodative vergence. Since the average total vergence in the viewing eye was less than one-half degree, about the size of the fixational fovea, this fixationholding mechanism was highly successful. Furthermore, maintenance of the target within the rod-free central fovea would keep the gain of the accommodative system at its maximum (Fincham, 1951; Campbell, 1954a, b; Phillips, 1974), thereby insuring sharp retinal imagery.

Acknowledgements-The authors are indebted to A. T. Bahill, E. Keller, and M. W. Morgan, Jr. for their helpful comments on earlier versions of the manuscript; to NIH Grant No. 5 T01 EY00076-04 and the Auxiliary to the American Optometric Association for financial support: and to Cynthia Cowee for assistance with the manuscript.

\section{REFERENCES}

Allen M. J. (1949) An objective high speed photographic technique for simultaneously recording changes in accommodation and convergence. Am. J. Oprom. and Archs Am. Acad. Optom. 26, 279-289.

Alpern M. and Ellen P. (1956) A quantitative analysis of the horizontal movements of the eyes in the experiment of Johannes Mueller-1. Methods and results. Am. J. Ophthal. 42, 289-303.

Bahill A. T., Clark M. R. and Stark L. (1975) Dynamic overshoot in saccadic eye movements is caused by neurological control signal reversals. Expl Neurol. 48. $107-122$.

Brodkey J. and Stark L. (1967) Accommodative convergence: and adaptive non-linear control system. Presbyterian-St. Luke's Hosp. Med. Bull. 6. 30-41.

Campbell F. W. (1954a) Accommodation reflex. Br. orthop. J. 11, 13-17.

Campbell F. W. (1954b) The minimum quantity of light required to elicit the accommodation refiex in man. $J$. Physiol., Lond. 123, 357-366.

Fincham E: F. (1951) The accommodation reflex and its stimulus. Br. J. Ophthal. 35, 381-393.

Hermann J. S. and Samson C. R. (1967) Critical detection of the accommodative convergence to accommodation ratio by photosensoroculography. Archs Ophthal. 78, 424-430.

Keller E. L. (1973) Accommodative vergence in the alert monkey: motor unit analysis. Vision Res. 13. 1565-1575.

Keller E. L. and Robinson D. A. (1972) Abducens unit behavior in the monkey during vergence movements. Vision Res. 12, 369-382.

Krishnan V. V.. Phillips S. and Stark L. (1973) Frequency analysis of accommodation, accommodative vergence and disparity vergence. Vision Res. 13, 1545-1554.

Müller J. (1826) Elements of Physiology, trans. by W. Baly (1843), Vol. 2, pp. 1147-1148. Taylor \& Walton. London.

Phillips S. R. (1974) Ocular neurological control systems; accommodation and the near response triad. Ph.D. dissertation, University of California. Department of Mechanical Engineering.

Pickwell L. D. (1972) Variation in ocular motor dominance. Br. J. physiol. Optics 27, 115-119.

Robinson D. A. (1966) The mechanism of human vergence eye movement. J. Ped. Ophthal. 3, 31-37.

Troeistra A., Zuber B. L., Miller D. and Stark L. (1964) Accommodative tracking: a trial-and-error function. Vision Res. 4, 585-594.

Yamamoto H. (1968) Studies on accommodative convergence. Jap. J. Ophthal. 12, 155-161.

Yamamoto H. (1970) Further studies on accommodative convergence. Jap. J. Ophthal. 14, 102-110. 